\title{
Carbon Nanotube Properties Influence Adsorption of Phenanthrene and Subsequent Bioavailability and Toxicity to Pseudokirchneriella subcapitata
}

\author{
Berit Glomstad, $^{\dagger}$ Dag Altin, ${ }^{\ddagger}$ Lisbet Sørensen, ${ }^{\S, \perp}$ Jingfu Liu, $^{\prime \prime}$ Bjørn M. Jenssen, ${ }^{\dagger}$ and Andy M. Booth*,§ \\ ${ }^{\dagger}$ Department of Biology, Norwegian University of Science and Technology, Trondheim NO-7491, Norway \\ ${ }^{\ddagger}$ BioTrix, Trondheim NO-7022, Norway \\ ${ }^{\S}$ SINTEF Materials and Chemistry, Trondheim NO-7465, Norway \\ "State Key Laboratory of Environmental Chemistry and Ecotoxicology, Research Center for Eco-Environmental Sciences, Chinese \\ Academy of Sciences, Beijing, 100085, China
}

\section{Supporting Information}

\begin{abstract}
The bioavailability of organic contaminants adsorbed to carbon nanotubes (CNTs) remains unclear, especially in complex natural freshwaters containing natural organic matter (NOM). Here, we report on the adsorption capacity $\left(Q^{0}\right)$ of five CNTs exhibiting different physicochemical properties, including a single-walled CNT (SWCNTs), multiwalled CNTs (MWCNT-15 and MWCNT-30), and functionalized MWCNTs (hydroxyl, $-\mathrm{OH}$, and carboxyl, $-\mathrm{COOH}$ ), for the model polycyclic aromatic hydrocarbon phenanthrene $(3.1-800 \mu \mathrm{g} / \mathrm{L})$. The influence of phenanthrene adsorption by the CNTs on bioavailability and toxicity was investigated using the freshwater algae Pseudokirchneriella subcapitata. CNTs were dispersed in algal growth media containing NOM (DOC, $8.77 \mathrm{mg} / \mathrm{L}$; dispersed concentrations: $0.5,1.3,1.3,3.3$, and $6.1 \mathrm{mg} / \mathrm{L}$ for

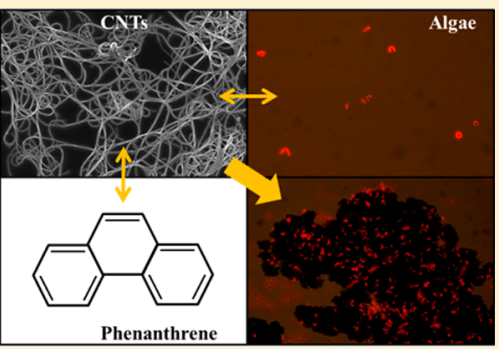
SWCNT, MWCNT-15, MWCNT-30, MWCNT-OH, and MWCNT-COOH, respectively). Adsorption isotherms of phenanthrene to the dispersed CNTs were fitted with the Dubinin-Ashtakhov model. $\mathrm{Q}^{0}$ differed among the CNTs, increasing with increasing surface area and decreasing with surface functionalization. SWCNT and MWCNT-COOH exhibited the highest and lowest $\log \mathrm{Q}^{0}(8.891$ and $7.636 \mu \mathrm{g} / \mathrm{kg}$, respectively). The presence of SWCNTs reduced phenanthrene toxicity to algae $\left(\mathrm{EC}_{50} ; 528.4\right)$ compared to phenanthrene-only $\left(\mathrm{EC}_{50} ; 438.3\right)$, and the presence of MWCNTs had no significant effect on phenanthrene toxicity. However, phenanthrene adsorbed to NOM-dispersed CNTs proved to be bioavailable and contribute to exert toxicity to P. subcapitata.
\end{abstract}

\section{INTRODUCTION}

The adsorption of hydrophobic organic compounds (HOCs) to carbon nanotubes (CNTs) and the subsequent influence on pollutant fate, bioavailability, and toxicity in aquatic systems is currently poorly understood. CNTs might act as a pollutant sink, reducing the bioavailability of HOCs through their adsorption. Alternatively, CNTs might increase the bioavailability of adsorbed pollutants through other uptake routes (e.g., ingestion) or by facilitating uptake (e.g., by affecting membrane integrity). Considering the strong sorption affinity of CNTs for HOCs, ${ }^{1-3}$ this interaction should be taken into account when assessing the environmental risk of CNTs. Polycyclic aromatic hydrocarbons (PAHs), which are main components in petrogenic oil and byproducts from incomplete combustion, are an environmentally ubiquitous class of priority pollutants. CNTs may be released into the environment as aggregates, composite particles, or dispersions. ${ }^{4}$ Adsorption of PAHs to CNTs has been previously investigated in several studies, ${ }^{1-3,5-8}$ and adsorption mechanisms of HOCs to CNTs have been extensively reviewed. ${ }^{9,10}$

Both the physicochemical properties of CNTs and environmental factors influence the adsorption of PAHs and other
HOCs to CNTs. CNT surface area appears to be positively related to adsorption capacity. ${ }^{3,10}$ In contrast, oxygen containing groups on the CNT surface have been reported to suppress the adsorption of HOCs due to an increase in polar regions unavailable for adsorption. ${ }^{11}$ Natural organic matter (NOM) present in natural waters will also reduce $\mathrm{PAH}$ adsorption through competition for available adsorption sites on the CNT surface. ${ }^{6,8,12}$ In addition to influencing adsorption, these factors are also likely to affect CNT fate in the environment, with oxygen functionalized CNTs and the presence of NOM reported to increase their aqueous dispersion concentration and stability. ${ }^{13-15} \mathrm{CNTs}$ with different physicochemical properties may, therefore, exhibit different potential for interaction with HOCs due to the specific behavior of CNT types in aqueous environments.

The toxicity of CNTs to aquatic organisms has been investigated in several studies. ${ }^{16-18}$ However, estimated

Received: October 21, 2015

Revised: December 18, 2015

Accepted: January 29, 2016

Published: January 29, 2016 
environmental concentrations of CNTs are likely to be below levels where toxic effects have been observed. ${ }^{19,20}$ At present, knowledge regarding the adsorption and cocontaminant toxicity of CNTs and HOCs in aquatic systems remains scarce and is often contradictory. CNT-bound diuron exhibited increased toxicity to the algae Chlorella vulgaris. ${ }^{21}$ In fish, PAHs adsorbed to CNTs have been reported to be both bioavailable ${ }^{22}$ and nonbioavailable. ${ }^{12}$ Phenanthrene adsorbed to $\mathrm{C}_{60}$ fullerene was bioavailable to the freshwater algae Pseudokirchneriella subcapitata and the zooplankton Daphnia magna $a^{23}$ and contributed to bioaccumulation but not to toxicity in the freshwater fish common carp (Cyprinus carpio). ${ }^{24}$ The presence of NOM has been suggested to impede desorption of PAHs from CNTs and thus affect bioavailability. ${ }^{12}$ It has also been reported that $\mathrm{CNT}$-adsorbed PAHs are bioavailable to both bacteria and benthic invertebrates. ${ }^{25,26}$ Despite the ubiquity of PAHs in the environment, the effect of CNT physicochemical properties on $\mathrm{PAH}$ toxicity to aquatic organisms has, to our knowledge, not been investigated. The use of NOM in studies regarding CNTs and cocontaminant toxicity is also scarce despite its known and possible effects on fate, adsorption, and toxicity.

In the present study, five CNT types, including one SWCNT, two pristine MWCNTs (MWCNT-15 and MWCNT-30), and two MWCNTs with oxygen-containing functional groups (MWCNT-OH and MWCNT-COOH) were dispersed in algal growth media containing NOM. Their adsorption of phenanthrene was studied, and adsorption isotherms were fitted to adsorption models used previously to describe HOC adsorption by CNTs. Finally, the effect of phenanthrene adsorption to CNTs on the subsequent bioavailability and toxicity of phenanthrene was investigated using P. subcapitata.

\section{MATERIALS AND METHODS}

Chemicals. A total of five types of carbon nanotubes (SWCNT, MWCNT-15, MWCNT-30, MWCNT-OH, and MWCNT-COOH (>95\%)) were purchased from Timesnano (Chengdu Organic Chemicals Co., Ltd., Chinese Academy of Sciences). Phenanthrene ( $\geq 99.5 \%)$ was purchased from SigmaAldrich. Suwannee River natural organic matter (SR-NOM) was supplied by the International Humic Substance Society (IHSS). All organic solvents, including methanol (Fluka Analytical), ethanol (VWR BDH Prolabo), dichloromethane (Rathburn Chemicals Ltd.), n-hexane (Fluka Analytical), and isopropanol (Lab Scan), were of analytical grade and tested for any impurities before use.

CNT Characterization. A summary of the CNT physicochemical properties supplied by the manufacturer is provided in Table S1. The CNTs were further characterized inhouse to verify their physicochemical properties (methods reported in the Supporting Information).

Dispersion and Exposure Media Preparation. SRNOM $(20 \mathrm{mg} / \mathrm{L})$ was dissolved in OECD algal growth media $(\text { TG201) })^{27}$ by magnetic stirring for $24 \mathrm{~h}$ and subsequently filtered through a $0.2 \mu \mathrm{m}$ Nalgene filter unit (Thermo Fisher Scientific, Inc.) to remove any undissolved material. After filtration, dissolved organic carbon (DOC) analysis (Sievers 900 TOC Analyzer, GE Analytical Instruments) showed a DOC concentration of $8.77 \pm 0.03 \mathrm{mg} / \mathrm{L}$. The solution $\mathrm{pH}$ was adjusted to $8.1 \pm 0.1$ using $\mathrm{NaOH}-\mathrm{HCl}$. TG201 containing SR-NOM, hereafter referred to as TG201-
NOM, was used for the preparation of all sample solutions and CNT dispersions throughout the study.

Preparation of CNT Dispersions. Aqueous CNT dispersions were prepared in a two-step procedure in which stock dispersions were prepared by bath sonication (Bandelin Sonorex Super RK 510H, 640W, $35 \mathrm{kHz}$ ) before being gently mixed into larger volumes of TG201-NOM $(1.8 \mathrm{~L})$ to achieve the desired initial concentration $(10 \mathrm{mg} / \mathrm{L})$. Sonication was performed by adding $50 \mathrm{~mL}$ of TG201-NOM into $250 \mathrm{~mL}$ Pyrex bottles containing the preweighed CNTs $(20 \pm 0.2 \mathrm{mg})$ followed by $15 \mathrm{~min}$ of sonication. This was repeated three more times until a final volume of $200 \mathrm{~mL}$ and a total sonication time of $1 \mathrm{~h}$ was reached. Further details of the CNT dispersion procedure are reported in the Supporting Information. The dispersions were left for $24 \mathrm{~h}$ to allow the settling of any undispersed CNTs, after which the supernatant was carefully removed by siphoning. To avoid dissimilarity in the treatment between the different CNT types, which could possibly influence adsorption behavior or affect toxicity, we implemented no further modification or adjustment of the CNT dispersions prior to use in the adsorption and toxicity experiments. As a result, the maximum environmental dispersion concentration for each of the CNT types was used in these studies.

CNT Dispersion Quantification. The final concentration of CNTs in the dispersions was determined by measuring absorption at $800 \mathrm{~nm}$ using a Hitachi U-2000 Spectrophotometer. Approximately $3 \mathrm{~mL}$ of each dispersion was pipetted into quartz cuvettes ( $3.5 \mathrm{~mL}$; Hellma Analytic) with a light path of $10 \mathrm{~mm}$. The measurements were conducted in duplicate. Measured absorbance was related to concentration through calibration curves prepared individually for each CNT (described in the Supporting Information). Calibration curves were in the range of $0.1-8 \mathrm{mg} / \mathrm{L} \mathrm{CNT}$, and all calibration curves had an $R^{2}$ value greater than 0.997 (Figure S1). The CNT concentrations in the dispersions used for adsorption and toxicity experiments were $0.5,1.3,1.3,3.3$, and $6.1 \mathrm{mg} / \mathrm{L}$ for SWCNT, MWCNT-15, MWCNT-30, MWCNT-OH, and MWCNT-COOH, respectively.

Adsorption of Phenanthrene to CNTs. Stock solutions of phenanthrene were prepared in methanol (1000, 100, and 10 $\mathrm{mg} / \mathrm{L})$ and then spiked into the prepared CNT dispersions at nominal concentrations $\left(C_{\text {nominal }}\right)$ ranging from 3.1 to $800 \mu \mathrm{g} /$ L. Aliquots $(20 \mathrm{~mL})$ of the phenanthrene-CNT dispersions in TG201-NOM were transferred to $22 \mathrm{~mL}$ glass vials (Agilent Technologies, Inc.) with PTFE lined caps. Samples were kept in the dark and left shaking on an orbital shaker (165 rpm) at room temperature for 5 days because previous studies ${ }^{3}$ have shown that this was sufficient for phenanthrene to equilibrate with CNTs. After 5 days, the samples were filtered through a hydrophilic polytetrafluoroethylene (PTFE) filter membrane ( $0.1 \mu \mathrm{m}$ pore size, Merck Millipore) to separate CNTs from the water phase. On the basis of measurements of absorbance, we determined that $>97 \%$ of the CNTs were removed by filtration (Table S2). Deuterated phenanthrene (d10) was added prior to filtration to account for any losses during filtration and the following extraction. Isopropanol $(5 \% \mathrm{v} / \mathrm{v})$ was added to each sample before being acidified $(<\mathrm{pH} 2)$ and stored at $4{ }^{\circ} \mathrm{C}$ until further handling. Phenanthrene was extracted using solid-phase extraction (SPE; Bond Elute PPL columns, Agilent Technologies, Inc.; details on the extraction method are available in the Supporting Information). The phenanthrene concentration in the water phase $\left(C_{\text {free }}\right)$ was determined using gas 
Table 1. Summary of the Physicochemical Properties of Each of the Five CNT Types Employed in the Study and Their Dispersion Concentration and Available Surface Area after 24 h of Settling ${ }^{a}$

\begin{tabular}{lcccccc}
\multicolumn{1}{c}{ CNT } & $\begin{array}{c}\text { average diameter } \\
(\mathrm{nm})\end{array}$ & $\begin{array}{c}\text { specific surface area } \\
\left(\mathrm{m}^{2} / \mathrm{g}\right)\end{array}$ & $\begin{array}{c}\text { surface oxygen } \\
\text { content }(\%)\end{array}$ & $\begin{array}{c}\text { surface carbon } \\
\text { content }(\%)\end{array}$ & $\begin{array}{c}\text { dispersion concentration } \\
(\mathrm{mg} / \mathrm{L})\end{array}$ & $\begin{array}{c}\text { available surface area } \\
\left(\mathrm{m}^{2} / \mathrm{L}\right)\end{array}$ \\
SWCNT & $2.4 \pm 1.1^{\mathrm{a}}$ & $483.7 \pm 23.5^{\mathrm{a}}$ & 1.6 & 98.4 & 0.5 & 0.26 \\
MWCNT-15 & $14.8 \pm 5.6^{\mathrm{b}}$ & $140.5 \pm 0.4^{\mathrm{b}}$ & 1.5 & 98.5 & 1.3 & 0.19 \\
MWCNT-30 & $16.6 \pm 8.6^{\mathrm{c}}$ & $177.4 \pm 3.0^{\mathrm{b}}$ & 2.0 & 98.0 & 1.3 & 0.23 \\
MWCNT-OH & $14.3 \pm 5.8^{\mathrm{b}}$ & $140.2 \pm 2.8^{\mathrm{b}}$ & 3.9 & 96.1 & 3.3 & 0.47 \\
MWCNT- & $20.3 \pm 7.5^{\mathrm{d}}$ & $139.7 \pm 3.5^{\mathrm{b}}$ & 5.7 & 94.3 & 6.1 & 0.86
\end{tabular}

${ }^{a}$ Variations are presented as standard deviations. Differing letters in the columns indicate statistically significant differences in average diameter and specific surface area among the CNTs (ANOVA: $p>0.05$ ).

chromatography-mass spectrometry (chemical analysis details are provided in the Supporting Information). All samples were conducted as full triplicates. $C_{\text {free }}$ was determined in the absence of CNTs in the concentration range 50-800 $\mu \mathrm{g} / \mathrm{L}$ to investigate for any loss of phenanthrene during the 5 days of shaking. Because this was negligible, the concentration of phenanthrene adsorbed to the CNTs $\left(\mathrm{C}_{\mathrm{CNT}}\right)$ was calculated from the measured $C_{\text {free }}$ and $C_{\text {nominal }}$.

A total of three sorption models previously used to describe the adsorption of PAHs to CNTs (the Langmuir model (LM), Freundlich model (FM), and the Dubinin-Ashtakhov (DAM) model (based on Polanyi theory) $)^{2,6-8,11}$ were fitted to the adsorption isotherms using GraphPad Prism 6.0 (GraphPad Software Inc.). The data were weighted by $1 / y^{2}$. The goodness of fit of the models was evaluated on the basis of the coefficient of determination $\left(R^{2}\right)$ and the mean weighted square error (MWSE). Model equations and parameters are described in Table S3.

CNT Influence on Phenanthrene Toxicity. CNT influence on phenanthrene toxicity to algae was evaluated using a modified OECD freshwater algae growth inhibition test. $^{27}$ The CNT TG201-NOM dispersions were prepared, spiked with phenanthrene $(50,100,200,400$, and $800 \mu \mathrm{g} / \mathrm{L})$ and then equilibrated for 5 days, as previously described. The phenanthrene $C_{\text {nominal }}$ range used was determined from a $72 \mathrm{~h}$ range finding test conducted with pure phenanthrene (no CNTs). After equilibration, the phenanthrene-CNT dispersions were inoculated with algae $\left(9.0 \pm 0.17 \times 10^{3}\right.$ cells $\left./ \mathrm{mL}\right)$ and transferred to a tilting table (Technomara Rockomat) under continuous illumination of $111 \pm 3.3 \mu \mathrm{E} / \mathrm{m}^{2} / \mathrm{s}$ (Philips TL-D 90 Graphics $18 \mathrm{~W} / 965)$ at a temperature of $20 \pm 2{ }^{\circ} \mathrm{C}$. Growth was determined after $0,24,48$, and $72 \mathrm{~h}$ by extracting $1 \mathrm{~mL}$ of each sample into $4 \mathrm{~mL}$ of ethanol (96\%) for $20 \mathrm{~min}$ and measuring fluorescence using a TD-700 fluorometer (excitation: $436 \mathrm{~nm}$, emission: $680 \mathrm{~nm}$; Turner Designs). This method is previously described ${ }^{28}$ and employed to assess algae growth in the presence of HOCs and $\mathrm{C}_{60}$ fullerene. ${ }^{23}$

To investigate the effect of CNTs on phenanthrene toxicity, we assessed the growth inhibition of algae caused by exposure to phenanthrene only with TG201-NOM-only samples (no phenanthrene) as controls. The $\mathrm{EC}_{50}$ concentrations determined served as the basis for measuring the changes in toxicity resulting from phenanthrene adsorption to CNTs. Algal growth in the controls was tested at three different time points over the study period to account for any variation. CNT-only dispersions without phenanthrene were used as controls to determine if there was any toxic contribution directly from the CNTs. A solvent control containing the highest amount of methanol used in the samples was included in the test. A total of four replicates at each test concentration and eight replicates of the controls were used. Sample $\mathrm{pH}$ was measured both at the beginning and at the end of the experiment. An increase in $\mathrm{pH}$ of two units in the controls was accepted as exponential growth was tested and observed throughout the experiment. Freely dissolved concentrations of phenanthrene at the end of the experiment $\left(C_{\text {free-end }}\right)$ were measured in samples with and without algae. Samples without algae showed negligible loss of phenanthrene during the 3 days of the toxicity study. Statistical difference between treatments throughout the study was investigated by analysis of variance (ANOVA) with Tukey's post hoc test in GraphPad Prism 6.0. The agglomeration of CNTs and algal cells was studied by fluorescent light microscopy (details presented in the Supporting Information).

\section{RESULTS AND DISCUSSION}

CNT Characterization. The determined physicochemical properties of the five CNT types employed in the study are summarized in Table 1. Measured diameters were generally consistent with those given by the manufacturer (Table S1), but there were large variations within the batches indicating heterogeneity. Specific surface areas (SSAs) determined in two different laboratories were very similar but differed from those reported by the manufacturer (Table S1). SWCNT exhibited a much higher SSA $\left(483.7 \mathrm{~m}^{2} / \mathrm{g}\right)$ than the MWCNTs. The surface oxygen content of MWCNT-OH and MWCNT$\mathrm{COOH}$ was $3.89 \%$ and $5.70 \%$, respectively. As expected, the surface oxygen content of the nonfunctionalized CNTs was lower (SWCNT, 1.64\%; MWCNT-15, 1.48\%; MWCNT-30, $1.96 \%)$ than on the functionalized CNTs. Further description of the CNTs based on transmission electron microscopy (TEM) is available in the Supporting Information.

CNT Dispersion. The five CNT types exhibited large differences in dispersibility. The percentage of the original 10 $\mathrm{mg} / \mathrm{L}$ of each CNT remaining in dispersion after the $24 \mathrm{~h}$ settling period was $61 \%$ MWCNT-COOH, 33\% MWCNTOH, 13\% MWCNT-15, 13\% MWCNT-30, and 5\% SWCNT. Final CNT dispersion concentrations and corresponding estimated SSAs in the samples are presented in Table 1. CNTs with polar oxygen functional groups exhibit a greater dispersibility than nonfunctionalized types. Furthermore, CNT diameter and SSA also appear to influence dispersibility, with SWCNTs having the smallest diameter and largest SSA yet exhibiting the lowest dispersibility of the CNTs studied. It should be noted that dispersion of CNTs using sonication might influence CNT properties, including SSA and surface chemistry. Bath sonication was selected over probe sonication in the current study to reduce the risk of CNT damage and also to increase the environmental relevance. ${ }^{1,8}$ However, these results are consistent with other studies reporting that CNT 
dispersibility and stability increases with surface oxygen content and diameter. ${ }^{14}$ Importantly, this data shows that different types of CNTs behave differently once released into aquatic environments, with longer water-column residence times for oxygen functionalized CNTs and quicker sedimentation for nonfunctionalized CNTs. SWCNTs appear more prone to rapid aggregation and sedimentation than the MWCNTs. Oxygen functionalized CNTs have the potential to be transported over greater distances, although this potential is dependent upon a number of other aquatic environmental parameters such as ionic strength, $\mathrm{pH},{ }^{29}$ and the presence of other particulate materials (heteroaggregation). ${ }^{30}$ From a toxicological perspective, this indicates that CNT properties could influence in which environmental compartments CNT exposure to organisms is more likely to occur (e.g., aqueous phase or sediments).

Adsorption of Phenanthrene to CNTs. Adsorption of HOCs by CNTs is considered to be nonlinear, even though linear adsorption of PAHs has been observed in low concentration ranges (pg-ng/L). ${ }^{2}$ Nonlinear adsorption models rather than a single adsorption coefficient $\left(K_{\mathrm{CNT}}\right)$ have, therefore, commonly been used to describe HOC adsorption by CNTs. ${ }^{9}$ The Langmuir model (LM), Freundlich model (FM), and the Dubinin-Ashtakhov model (DAM) (based on the Polanyi theory) have all been previously used to describe HOC adsorption, including PAHs, by CNTs. ${ }^{2,6-8,11}$ Adsorption isotherms of phenanthrene to the five CNT types and the DAM fitted curves are presented in Figure 1, and fitted

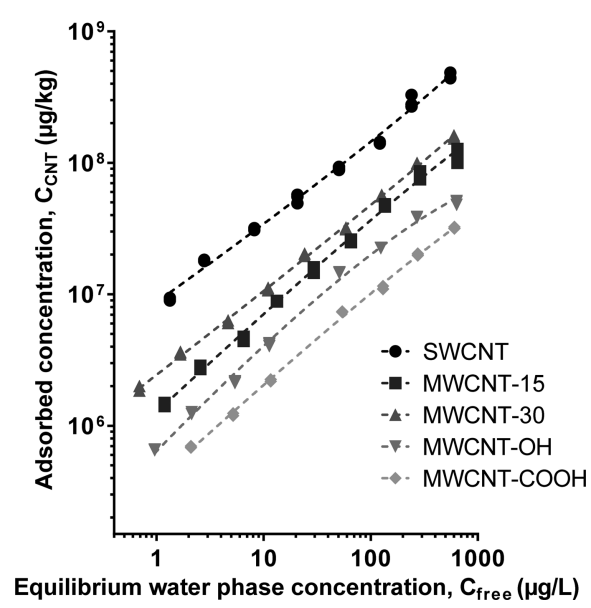

Figure 1. Adsorption isotherms of phenanthrene to five types of CNTs, plotted as individual replicates. The Dubinin-Ashtakhov model fitted the experimental data well. SWCNTs exhibited higher adsorption capacity than the MWCNTs, and a lower adsorption capacity was observed for functionalized MWCNTs compared to nonfunctionalized MWCNTs.

parameters are given in Table S4. Fitted curves and model parameters of the LM and FM are available in Figure S2 and Table S4. In the present study, both the FM and DAM fitted the data well, while the LM did not seem suitable for describing the adsorption of phenanthrene to CNTs. The criteria for evaluating the goodness of fit ( $R^{2}$ and MWSE) showed slightly better fit of the DAM than the FM. In addition, DAM has previously proven to describe the adsorption of PAHs to CNTs well under various conditions, e.g., in the presence of humic acid (HA) and different dispersion states. ${ }^{8}$ Further discussions will therefore focus on the DAM parameters.
The DAM parameters allow the evaluation of adsorption in terms of adsorption capacity $\left(Q^{0}\right)$ and adsorption affinity, identified by $E$ (Table S3). Here, $b$ is a fitting parameter (although $b$ has also been found to be related to adsorption affinity). ${ }^{11} Q^{0}$ depends on the available adsorption sites on the CNTs, representing the maximum amount of a specific HOC that can be adsorbed. Adsorption affinity describes the strength of the interactions between the CNTs and the HOC. ${ }^{10} \mathrm{~A}$ thorough discussion on the DAM and its parameters can be found in the review by Yang and Xing. ${ }^{10}$ In the adsorption isotherms, the adsorbed mass of phenanthrene is normalized against CNT mass. This allows the adsorption behavior of CNTs to be directly compared despite the observed differences in dispersed concentrations. A recent study also showed that adsorption isotherms of the PAH fluoranthene were similar at varying MWCNT concentrations. ${ }^{12}$

$Q^{0}$ decreased significantly in the order SWCNT $>$ MWCNT$30>$ MWCNT-15 > MWCNT-OH $>$ MWCNT-COOH $(p$ $<0.013)$. $Q^{0}$ of SWCNT was 4.7 and 3.2 times higher than those of MWCNT-15 and MWCNT-30, respectively. This is likely to be due to the higher SSA of SWCNT, indicating the important role SSA has in controlling adsorption capacity. This is consistent with a previous review of existing adsorption data, which showed a linear relationship between $Q^{0}$ for phenanthrene by CNTs and CNT SSA. ${ }^{10}$ Although MWCNT-OH and MWCNT-COOH have similar SSAs as MWCNT-15, they both exhibit a significantly lower $Q^{0}$ for phenanthrene. Again, this is in accordance with previous studies showing that increased surface oxygen content is also directly related to a decrease in $Q^{0}$. The presence of oxygen-containing functional groups reduces the number of potential adsorption sites for hydrophobic HOCs and increases the overall hydrophilicity of the CNTs. ${ }^{10}$ The decrease in $Q^{0}$ has also been explained by competition from water molecules for available adsorption sites at the functional groups. ${ }^{11}$

To create an environmentally relevant test system, CNT dispersions were prepared in the presence of $20 \mathrm{mg} / \mathrm{L}$ (nominal) NOM prior to addition of the phenanthrene. Despite the use of a filtration step $(0.2 \mu \mathrm{m})$ to remove any particulate NOM from the aqueous phase, nanosized NOM may still have been present, possibly offering competing adsorption sites for the dissolved phenanthrene. A prestudy indicated no adsorption of phenanthrene by SR-NOM itself (data not shown), which is consistent with recent observations made regarding the effect of SR-NOM on fluoranthene bioavailability. ${ }^{12}$ However, the dispersion preparation settling step offers a $24 \mathrm{~h}$ period for NOM adsorption to the CNTs prior to the addition of dissolved phenanthrene to the system. As a result, phenanthrene adsorption is occurring (at least partially) to NOM-coated CNTs. Directly evaluating the effects of NOM on pollutant adsorption was beyond the scope of this study but has been previously investigated. ${ }^{6,8}$ In these studies, NOM has been observed to aid CNT dispersion, increase CNT SSA available for pollutant adsorption through a reduction in CNT aggregation, and increase adsorption isotherm linearity. However, the overriding influence of NOM appears to be a decrease in CNT adsorption capacity for pollutants arising from competition for available adsorption sites. ${ }^{8}$ Importantly, the phenanthrene adsorption data generated in the present study for the five CNTs continues to follow established HOC-CNT adsorption patterns despite the presence of NOM.

Due to differences in dispersibility, each CNT type exhibits a different maximum environmental dispersion concentration. As 
a result, the quantity of phenanthrene adsorbed and the quantity remaining freely dissolved in the water phase does not reflect the individual $\mathrm{CNT}$ adsorption capacity. The percentage of $C_{\text {nominal }}$ phenanthrene adsorbed to each CNT type at different concentrations is presented in Figure 2. SWCNTs

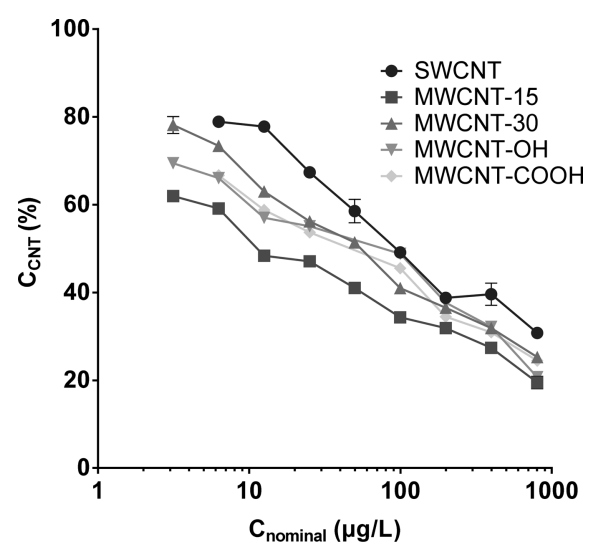

Figure 2. Percentage of phenanthrene adsorbed to CNTs at different phenanthrene concentrations. Error bars represent the standard error of mean. The pattern showed the highest percentage adsorbed to SWCNT and lowest to MWCNT- $\mathrm{COOH}$, although not significant at all $C_{\text {nominal }}$ values.

adsorbed the highest quantity of phenanthrene at all tested concentrations, while MWCNT-15 adsorbed the lowest quantity. However, these patterns were not statistically significant at all phenanthrene concentrations. No pattern was observed for the other CNTs. The dispersion concentration of the nonfunctionalized MWCNTs was approximately 2 times higher than that of SWCNT, whereas for MWCNT-COOH, it was more than 1 order of magnitude higher than that of SWCNT. Although SWCNTs have a low dispersibility, their high adsorption capacity means they appear to exhibit an adsorption effect on cocontaminants that is similar to other more highly dispersible CNTs. If representatives from this suite of CNTs are present in similar concentrations (e.g., below the maximum environmental concentration), significantly different quantities of HOCs would be correspondingly adsorbed to the different CNTs. The present study highlights the necessity of determining key physicochemical properties of CNTs when evaluating their potential environmental behavior and impacts. Importantly, the results show that the common approach of classifying all CNT types as a single group or nanomaterial type with an assumed equal environmental behavior would be incorrect.

Conducting a meaningful comparison of data generated in different studies on CNTs is often challenging due to differences in experimental approaches employed (e.g., exposure solutions, use or absence of NOM, and dispersion preparation techniques). However, these very differences can help to explain certain observations. Compared with previous studies addressing phenanthrene adsorption to CNTs, we typically observe a higher $Q^{0}$ (comparing nonfunctionalized MWCNTs). ${ }^{2,7}$ The present study employed sonication for CNT dispersion, whereas many other studies have mixed phenanthrene with CNTs without dispersing the CNTs beforehand. ${ }^{2,3,7}$ Sonication has been shown to increase CNT adsorption capacity to pyrene and was attributed to an increase in the exposed CNT SSA after sonication. ${ }^{8}$ The present study used only CNTs that remained dispersed after the $24 \mathrm{~h}$ settling period, indicating that most CNTs in the adsorption studies were well dispersed and that CNT aggregates with lower SSA were not present. The high CNT adsorption capacity observed in this study, even in the presence of NOM, indicates that dispersion preparation method is a key parameter to consider when evaluating CNT adsorption of HOCs. The effect of sonication on adsorption was found to be irreversible even after reaggregation of the $\mathrm{CNTs},{ }^{8}$ which might be of environmental relevance as sonication can be used in CNT pretreatment for various applications. ${ }^{4}$

No clear relationship was observed between $E$ and either CNT SSA or surface chemistry, indicating that these parameters do not exert a significant influence on phenanthrene interaction and adsorptive strength. Although it has been previously suggested that the presence of surface oxygen containing groups on CNTs can increase $E,{ }^{31}$ our data are in accordance with recent observations, showing no effect of surface oxidation on $E .^{11}$ A thorough discussion on the effect of CNT surface chemistry on the mechanisms of interaction and their strength has previously been presented. ${ }^{11}$ The presence of NOM is, however, likely to decrease $E,{ }^{8,10}$ and this may be the reason why lower $E$ values were observed in the present study compared to previous studies. ${ }^{2,7}$

Toxicity of CNTs. CNT-only samples were studied at single concentrations (maximum environmental concentrations: SWCNT, 0.5; MWCNT-15, 1.3; MWCNT-30, 1.3; MWCNT-OH, 3.3; and MWCNT-COOH, $6.1 \mathrm{mg} / \mathrm{L})$. Thus, no $\mathrm{EC}_{50}$ value could be established. However, the effect of CNTs on algal growth was determined by comparing average specific growth rates in the CNT only samples with those in the control samples. When all control samples and CNT-only samples were treated as a single group, the coefficient of variation $(\mathrm{CV})$ of average specific algal growth rate was $3.76 \%$. When all control samples and specific CNT-only samples were treated as a single group, the CV did not exceed $4.09 \%$ (MWCNT-COOH) for any CNT type. All CV values were below the recommended threshold of $7 \%,{ }^{27}$ showing that the effect of CNT only dispersions on algal growth was negligible.

Phenanthrene Toxicity in the Absence and Presence of CNTs. Growth rates of $P$. subcapitata exposed to $C_{\text {nominal }}$ phenanthrene-only and phenanthrene in mixtures with CNTs (at maximum environmental concentrations) are shown in Figure 3. To account for any differences between the CNTs, we

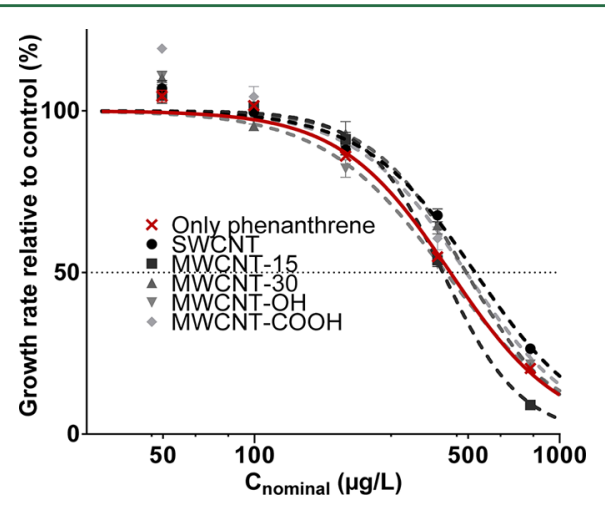

Figure 3. Relative growth rate of $P$. subcapitata exposed to increasing nominal phenanthrene concentrations in the absence (red) and present (gray) of CNT. Error bars represent the standard error of mean. A significant effect of the presence of CNTs compared to phenanthrene-only was only observed for SWCNT. 


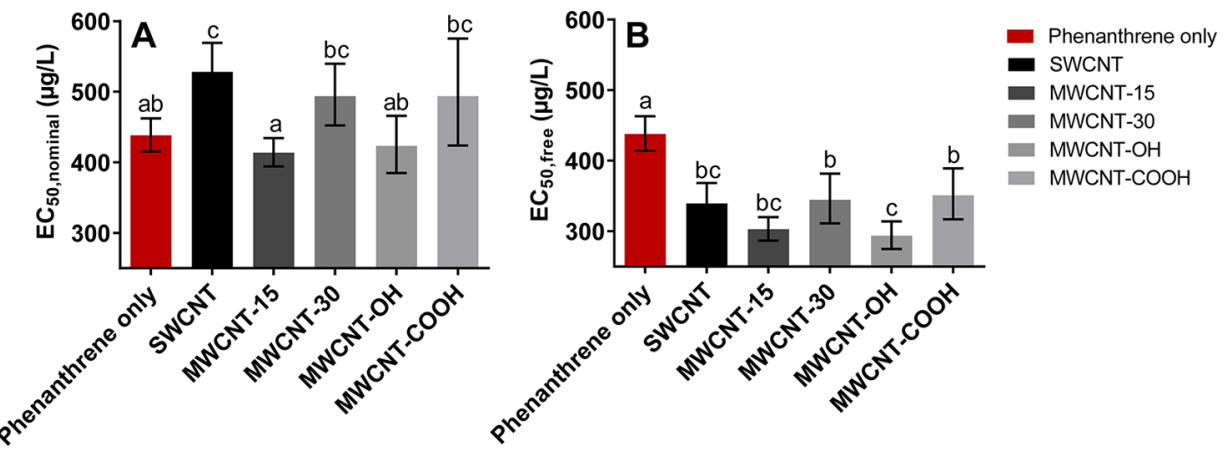

Figure 4. $\mathrm{EC}_{50}$ values calculated based on $(\mathrm{A})$ the nominal phenanthrene concentration $\left(C_{\text {nominal }}\right)$ and $(\mathrm{B})$ the phenanthrene concentrations in the water phase $\left(C_{\text {free }}\right)$. Error bars show the $95 \%$ confidence interval. Common letters above the bars indicate no significant difference between groups $(p$ $>0.05)$.

normalized growth curves against the respective CNT control. Because the $\mathrm{CV}$ in the controls without phenanthrene (a total of 24 replicates over 41 days) was $4.04 \%$, variation in algal growth was not considered to be significant. Solvent control samples showed no significant toxicity resulting from methanol (CV: $4.42 \%$ ). The phenanthrene-only concentration range caused $0-80 \%$ inhibition of algal growth rate, meeting with the criteria of the test guidelines. By the end of the experiment, $\mathrm{pH}$ had decreased (not consistently) below the accepted limit at high phenanthrene concentrations in combined CNTphenanthrene exposures but not in controls containing CNTs only or phenanthrene only. No pattern in reduced or enhanced growth or effect on the shape of the growth curves was observed.

$C_{\text {nominal }}$ and $C_{\text {free }}$ values at which $50 \%$ growth inhibition occurred $\left(\mathrm{EC}_{50}\right)$ are presented in Figure 4 and listed in Table S5. Phenanthrene-only exposure resulted in an $\mathrm{EC}_{50}$ value of $438.3 \mu \mathrm{g} / \mathrm{L}$. Although this value is lower than that reported for a $48 \mathrm{~h}$ growth inhibition test, ${ }^{23}$ it is expected given the use of a $72 \mathrm{~h}$ exposure time in the present study. Growth stimulation was observed at the lowest concentration of phenanthrene (50 $\mu \mathrm{g} / \mathrm{L}$ ), both in the absence and in the presence of CNTs. This is a phenomena observed in several other studies with exposure of algae to low concentrations of $\mathrm{PAH} .{ }^{32,33}$

When $\mathrm{EC}_{50}$ values based on $C_{\text {nominal }}$ are considered, the presence of CNTs did not show a uniform effect (increase or decrease) on phenanthrene toxicity to $P$. subcapitata (Figure 4A). The adsorption study showed that at the phenanthrene concentrations used in the present toxicity study $(50-800 \mu \mathrm{g} /$ $\mathrm{L})$, between $\sim 20 \%$ and $\sim 60 \%$ of $C_{\text {nominal }}$ phenanthrene was adsorbed to the CNTs (Figure 2). However, a significant decrease in $\mathrm{CNT}$-phenanthrene toxicity compared to phenanthrene-only was just observed for SWCNT, where the $\mathrm{EC}_{50}$ increased to $528.4(p=0.016)$. MWCNT-30 and MWCNT$\mathrm{COOH}$ exhibited a nonsignificant reduction in toxicity, and MWCNT-15 and MWCNT-OH EC 50 exhibited a nonsignificant increase in toxicity. Even though a substantial proportion of $C_{\text {nominal }}$ was adsorbed to the CNTs, no significant effect (reduction or increase) on algal toxicity could be observed for four of the five CNTs. Similar results have previously been reported for $C$. vulgaris, where the presence of $10 \mathrm{mg} / \mathrm{L}$ pristine or oxidized CNTs had no effect on diuron toxicity to the algae, determined on the basis of $C_{\text {nominal. }}$

To further investigate the importance of the CNT-adsorbed versus $C_{\text {free }}$ phenanthrene, we calculated $\mathrm{EC}_{50}$ values based on $C_{\text {free }}$ at the start of the exposure, $\mathrm{EC}_{50 \text {,free }}$ (Figure 4B, Table S5). Compared to $\mathrm{EC}_{50 \text {,free }}$ for phenanthrene-only $(438.0 \mu \mathrm{g} / \mathrm{L})$,
$\mathrm{CNT} /$ phenanthrene $\mathrm{EC}_{50, \text { free }}$ values were significantly reduced $(p<0.0015)$ for all CNTs, ranging from $293.8 \mu \mathrm{g} / \mathrm{L}$ (MWCNT-OH) to $351.2 \mu \mathrm{g} / \mathrm{L}$ (MWCNT-COOH) (Table S5). This corresponds to an apparent increase in phenanthrene toxicity of between $20 \%$ and $33 \%$ in the presence of the CNTs and, importantly, suggests that the CNT-adsorbed phenanthrene is at least partly bioavailable and contributes to the total toxicity. Although no toxicity was observed for any of the CNTs, synergistic effects resulting from the interaction between phenanthrene and the CNTs could occur and contribute to the apparent increase in toxicity. Phenanthrene desorption from CNTs due to the influence of algae on $C_{\text {free }}$ may also contribute to toxicity (discussed further in the following paragraph). However, these data support previous findings that CNMadsorbed HOCs are bioavailable for uptake in algae. ${ }^{21,23} \mathrm{C}_{60}$ fullerene increased phenanthrene toxicity to $P$. subcapitata due to direct phenanthrene transport to the cell membrane. ${ }^{23}$ Diuron toxicity to algae increased in the presence of CNTs due to increased exposure concentration in the boundary layer of algae in contact with CNT aggregates. ${ }^{21}$ In the present study, fluorescent light microscopy images revealed significant agglomeration of CNT aggregates and algal cells (Figure 5). It is therefore probable that the attachment of algae to CNT aggregates facilitates the transport and bioavailability of CNTadsorbed phenanthrene to the algal cell membranes. Furthermore, relative exposure concentrations may appear higher for CNT-adsorbed phenanthrene compared to phenanthrene present only in the dissolved phase.

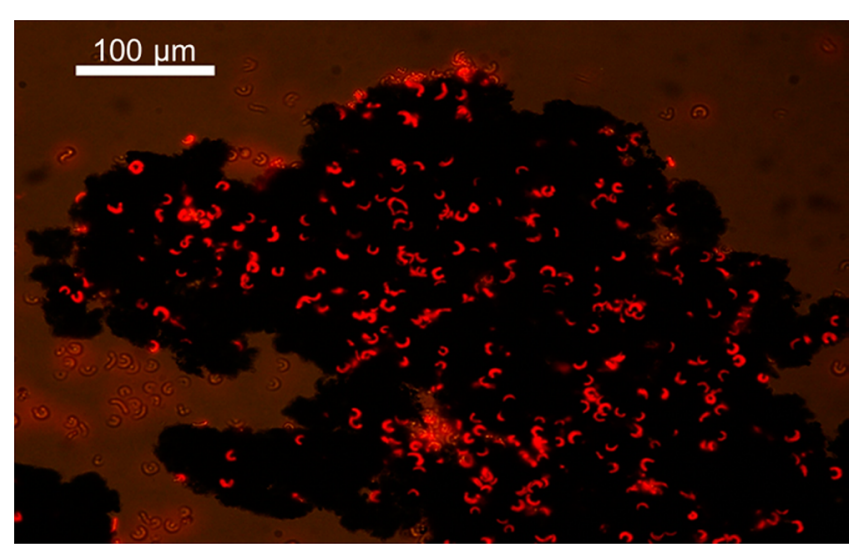

Figure 5. Fluorescent light microscopy image of CNT aggregate with algal cells attached. The red color of the algal cells is due to fluorescence from chlorophyll. (Photo: Dag Altin, BioTrix). 
Phenanthrene concentration in the water phase was determined at the end of the experiment $\left(C_{\text {free-end }}\right)$ for phenanthrene-only samples in both the presence and absence of algae (Figure S3). Differences in $C_{\text {free-end }}$ in the presence and absence of algae serve as a measure of phenanthrene association with algae (e.g., uptake, sorption). At the lowest $C_{\text {nominal }}$ (50 $\mu \mathrm{g} / \mathrm{L}), C_{\text {free-end }}$ was $61 \%$ lower in the presence of algae compared to in absence of algae. At higher $C_{\text {nominal }}$ values (100, $200,400$, and $800 \mu \mathrm{g} / \mathrm{L}), C_{\text {free-end }}$ was $56 \%, 39 \%, 14 \%$, and $8 \%$ lower, respectively. This shows the algae reduces $C_{\text {free, }}$ particularly at lower $C_{\text {nominal }}$ values, thus altering system equilibrium. Phenanthrene adsorption by CNTs in water is reversible, ${ }^{34}$ so a decrease in $C_{\text {free }}$ due to association with algae might promote phenanthrene desorption from CNTs as a new equilibrium between $C_{\text {free }}$ and $C_{\mathrm{CNT}}$ is established. In addition to altered exposure routes and concentrations resulting from algae-CNT attachment, phenanthrene desorption from CNTs may, therefore, also contribute to the increased toxicity observed in the present study. Here, CNTs could function as passive dosing materials similar to those employed to control HOC exposure in aquatic toxicity tests. ${ }^{35}$ Further assessment of $\mathrm{PAH}$ desorption from CNTs would be interesting from both an ecotoxicological and a technical perspective.

A pair of recent studies investigating CNT-adsorbed $\mathrm{PAH}$ bioavailability showed contradictory findings considered to result from the presence and absence of NOM. SWCNTadsorbed phenanthrene was bioavailable to Japanese medaka (Oryzias latipes) $^{22}$ and MWCNT-adsorbed fluoranthene was not bioavailable to fathead minnow (Pimephales promelas) when NOM was present. ${ }^{12}$ It was suggested that NOM suppresses fluoranthene desorption from CNTs, reducing the bioavailability of the adsorbed fraction to fish. ${ }^{12}$ In the present study, phenanthrene toxicity in the presence of CNTs without NOM was not specifically investigated. However, CNT-adsorbed phenanthrene was clearly bioavailable to algae in the presence of NOM for all five CNTs studied. This contradicts the findings of the previous study, ${ }^{12}$ indicating further work is needed to fully understand the complex interplay between CNTcocontaminant interaction and toxicity in the presence of NOM.

An aim of this study was to investigate the role of CNT physicochemical properties on phenanthrene bioavailability and toxicity. In a comparison of the CNTs at their maximum environmental concentrations, phenanthrene toxicity was significantly higher in the presence of MWCNT-15 compared to SWCNT, MWCNT-30, and MWCNT-COOH and for MWCNT-OH compared to SWCNT $(p<0.042)$. However, no direct correlation was observed between phenanthrene toxicity $\left(\mathrm{EC}_{50 \text {,nominal }}\right)$ and CNT SSA, surface chemistry (oxygen content), or CNT concentration (Figure S4A-C). Nevertheless, a positive correlation between adsorption capacity normalized against CNT mass $\left[Q^{0}(\mu \mathrm{g} / \mathrm{kg}) \times\right.$ mass CNT in samples $(\mathrm{kg})]$ and $\mathrm{EC}_{50 \text {,nominal }}$ was observed (Figure S5, Pearson correlation $\left.R^{2}=0.8075, p=0.038\right)$. Although phenanthrene toxicity in the present study cannot be directly related to individual CNT properties, the adsorption capacity, which is dependent on several CNT properties, appears to be a key parameter for CNT-cocontaminant toxicity.

If the observed increase in toxicity based on $C_{\text {free }}$ is related to transport of phenanthrene to cell membranes and increased exposure concentrations, then variation in $\mathrm{EC}_{50 \text {,free }}$ concentrations could be expected on the basis of the determined differences in $C_{\mathrm{CNT}}$. However, a significant difference in $\mathrm{EC}_{50 \text {,free }}$ was only observed for MWCNT-OH compared to MWCNT$30(p=0.047)$ and MWCNT-COOH $(p=0.026)$. Comparable $\mathrm{EC}_{50 \text {,free }}$ values indicate that the toxicity contribution from adsorbed phenanthrene is similar for the CNTs tested. This similarity may be a result of the differences in CNT dispersion concentrations. While $C_{\mathrm{CNT}}$ is highest for SWCNT, the dispersion concentration is low. Conversely, $C_{\mathrm{CNT}}$ is low for $\mathrm{MWCNT}-\mathrm{COOH}$, but the dispersion concentration is high, possibly leading to increased $\mathrm{CNT}$-algae attachment and phenanthrene exposure. Further studies are needed to fully understand the contribution of CNT-adsorbed phenanthrene to algal toxicity under environmentally relevant conditions.

The present study highlights the importance of CNT physicochemical properties on their environmental fate, adsorption of cocontaminants and their effect on cocontaminant toxicity. The investigation of $\mathrm{CNT}$-cocontaminant interaction and toxicity in the presence of NOM provides an environmentally relevant test scenario few other studies have addressed previously. Importantly, we have shown that CNTs cannot be considered a sink for HOCs as these remain bioavailable and can contribute to toxicity, possibly through alternative uptake routes. NOM clearly plays a critical role in controlling the adsorption, bioavailability, and toxicity of HOCs in the presence of CNTs and requires further study for a complete understanding.

\section{ASSOCIATED CONTENT}

\section{Supporting Information}

The Supporting Information is available free of charge on the ACS Publications website at DOI: 10.1021/acs.est.5b05177.

Additional details on CNT physicochemical characterization, preparation of CNT dispersions and calibration curves, solid-phase extraction for the extraction of phenanthrene from CNT/TG201-NOM dispersions, chemical analysis of phenanthrene extracts, microscopic analysis of CNT-algae interaction, and qualitative description of the carbon nanotubes. Tables showing CNT physicochemical properties supplied by the manufacturer, measurements of UV-vis absorbance at $800 \mathrm{~nm}$ in CNT dispersions before and after filtration through a $0.1 \mu \mathrm{m}$ polytetrafluoroethylene filter membrane, adsorption-model equations and description of their parameters, fitted parameters of models for adsorption isotherms of phenanthrene to five types of CNTs, and concentration at which 50\% growth inhibition of $P$. subcapitata occurred when exposed to phenanthrene and to phenanthrene in a mixture with CNTs based on the nominal concentration of phenanthrene and on phenanthrene concentrations in the water phase. Figures showing CNT calibration curves determined by UV-vis spectroscopy at $800 \mathrm{~nm}$, adsorption isotherms fitted to models, and phenanthrene concentration in the water phase in samples containing only phenanthrene at the end of the experiment in the presence and absence of algae. (PDF)

\section{AUTHOR INFORMATION}

\section{Corresponding Author}

*Tel.: +47 93089510; e-mail address: andy.booth@sintef.no.

\section{Present Address}

${ }^{\perp}$ Institute of Marine Research, Bergen NO-5817, Norway 


\section{Notes}

The authors declare no competing financial interest.

\section{ACKNOWLEDGMENTS}

The work reported here has been undertaken as part of the Research Council of Norway (RCN) funded project "NanoSorb” (Grant Agreement number 209685/E50). The authors wish to thank the RCN for their financial support. We also wish to thank the External Cooperation Program of Chinese Academy of Sciences (Grant number GJHZ1206) for financial support. The authors acknowledge the essential technical assistance of Kristin Bonaunet, Lisbet Støen, Inger Steinsvik, Marianne Rønsberg, Kjersti Almås, Calin D. Marioara, John Walmsley and Aud Spjelkavik (SINTEF Materials and Chemistry). We would also like to thank Gurvinder Singh for creating the SEM images used for characterizing the CNT materials.

\section{REFERENCES}

(1) Kah, M.; Zhang, X. R.; Hofmann, T. Sorption behavior of carbon nanotubes: Changes induced by functionalization, sonication and natural organic matter. Sci. Total Environ. 2014, 497, 133-138.

(2) Kah, M.; Zhang, X.; Jonker, M. T. O.; Hofmann, T. Measuring and Modelling Adsorption of PAHs to Carbon Nanotubes Over a Six Order of Magnitude Wide Concentration Range. Environ. Sci. Technol. 2011, 45 (14), 6011-6017.

(3) Yang, K.; Zhu, L. Z.; Xing, B. S. Adsorption of polycyclic aromatic hydrocarbons by carbon nanomaterials. Environ. Sci. Technol. 2006, 40 (6), 1855-1861.

(4) Kohler, A. R.; Som, C.; Helland, A.; Gottschalk, F. Studying the potential release of carbon nanotubes throughout the application life cycle. J. Cleaner Prod. 2008, 16 (8-9), 927-937.

(5) Hou, L.; Zhu, D.; Wang, X.; Wang, L.; Zhang, C.; Chen, W. Adsorption of phenanthrene, 2-naphthol, and 1-naphthylamine to colloidal oxidized multiwalled carbon nanotubes: Effects of humic acid and surfactant modification. Environ. Toxicol. Chem. 2013, 32 (3), 493-500.

(6) Wang, X.; Tao, S.; Xing, B. Sorption and Competition of Aromatic Compounds and Humic Acid on Multiwalled Carbon Nanotubes. Environ. Sci. Technol. 2009, 43 (16), 6214-6219.

(7) Yang, K.; Wang, X.; Zhu, L.; Xing, B. Competitive sorption of pyrene, phenanthrene, and naphthalene on multiwalled carbon nanotubes. Environ. Sci. Technol. 2006, 40 (18), 5804-5810.

(8) Zhang, X.; Kah, M.; Jonker, M. T. O.; Hofmann, T. Dispersion State and Humic Acids Concentration-Dependent Sorption of Pyrene to Carbon Nanotubes. Environ. Sci. Technol. 2012, 46 (13), 71667173.

(9) Pan, B.; Xing, B. Adsorption Mechanisms of Organic Chemicals on Carbon Nanotubes. Environ. Sci. Technol. 2008, 42 (24), 90059013.

(10) Yang, K.; Xing, B. Adsorption of Organic Compounds by Carbon Nanomaterials in Aqueous Phase: Polanyi Theory and Its Application. Chem. Rev. 2010, 110 (10), 5989-6008.

(11) Wu, W. H.; Chen, W.; Lin, D. H.; Yang, K. Influence of Surface Oxidation of Multiwalled Carbon Nanotubes on the Adsorption Affinity and Capacity of Polar and Nonpolar Organic Compounds in Aqueous Phase. Environ. Sci. Technol. 2012, 46 (10), 5446-5454.

(12) Linard, E. N.; van den Hurk, P.; Karanfil, T.; Apul, O. G.; Klaine, S. J. Influence of carbon nanotubes on the bioavailability of fluoranthene. Environ. Toxicol. Chem. 2015, 34 (3), 658-666.

(13) Hyung, H.; Fortner, J. D.; Hughes, J. B.; Kim, J. H. Natural organic matter stabilizes carbon nanotubes in the aqueous phase. Environ. Sci. Technol. 2007, 41 (1), 179-184.

(14) Schwyzer, I.; Kaegi, R.; Sigg, L.; Smajda, R.; Magrez, A.; Nowack, B. Long-term colloidal stability of 10 carbon nanotube types in the absence/presence of humic acid and calcium. Environ. Pollut. 2012, 169, 64-73.
(15) Shen, M.-H.; Yin, Y.-G.; Booth, A.; Liu, J.-F. Effects of molecular weight-dependent physicochemical heterogeneity of natural organic matter on the aggregation of fullerene nanoparticles in mono- and divalent electrolyte solutions. Water Res. 2015, 71 (0), 11-20.

(16) Alloy, M. M.; Roberts, A. P. Effects of suspended multi-walled carbon nanotubes on daphnid growth and reproduction. Ecotoxicol. Environ. Saf. 2011, 74 (7), 1839-1843.

(17) Schwab, F.; Bucheli, T. D.; Lukhele, L. P.; Magrez, A.; Nowack, B.; Sigg, L.; Knauer, K. Are Carbon Nanotube Effects on Green Algae Caused by Shading and Agglomeration? Environ. Sci. Technol. 2011, 45 (14), 6136-6144.

(18) Edgington, A. J.; Roberts, A. P.; Taylor, L. M.; Alloy, M. M.; Reppert, J.; Rao, A. M.; Mao, J. D.; Klaine, S. J. The Influence of natural organic matter on the toxicity of multiwalled carbon nanotubes. Environ. Toxicol. Chem. 2010, 29 (11), 2511-2518.

(19) Mueller, N. C.; Nowack, B. Exposure Modeling of Engineered Nanoparticles in the Environment. Environ. Sci. Technol. 2008, 42 (12), 4447-4453.

(20) Gottschalk, F.; Lassen, C.; Kjoelholt, J.; Christensen, F.; Nowack, B. Modeling Flows and Concentrations of Nine Engineered Nanomaterials in the Danish Environment. Int. J. Environ. Res. Public Health 2015, 12 (5), 5581-5602.

(21) Schwab, F.; Bucheli, T. D.; Camenzuli, L.; Magrez, A.; Knauer, K.; Sigg, L.; Nowack, B. Diuron Sorbed to Carbon Nanotubes Exhibits Enhanced Toxicity to Chlorella vulgaris. Environ. Sci. Technol. 2013, 47 (13), 7012-7019.

(22) Su, Y.; Yan, X. M.; Pu, Y. B.; Xiao, F.; Wang, D. S.; Yang, M. Risks of Single-Walled Carbon Nanotubes Acting as ContaminantsCarriers: Potential Release of Phenanthrene in Japanese Medaka (Oryzias latipes). Environ. Sci. Technol. 2013, 47 (9), 4704-4710.

(23) Baun, A.; Sorensen, S. N.; Rasmussen, R. F.; Hartmann, N. B.; Koch, C. B. Toxicity and bioaccumulation of xenobiotic organic compounds in the presence of aqueous suspensions of aggregates of nano-C-60. Aquat. Toxicol. 2008, 86 (3), 379-387.

(24) Hu, X. L.; Li, J.; Shen, M. H.; Yin, D. Q. Fullerene-associated phenanthrene contributes to bioaccumulation but is not toxic to fish. Environ. Toxicol. Chem. 2015, 34 (5), 1023-1030.

(25) Shen, M.; Xia, X.; Zhai, Y.; Zhang, X.; Zhao, X.; Zhang, P. Influence of carbon nanotubes with preloaded and coexisting dissolved organic matter on the bioaccumulation of polycyclic aromatic hydrocarbons to Chironomus plumosus larvae in sediment. Environ. Toxicol. Chem. 2014, 33 (1), 182-189.

(26) Shen, M. H.; Xia, X. H.; Wang, F.; Zhang, P.; Zhao, X. L. Influences of multiwalled carbon nanotubes and plant residue chars on bioaccumulation of polycyclic aromatic hydrocarbons by Chironomus plumosus larvae in sediment. Environ. Toxicol. Chem. 2012, 31 (1), 202-209.

(27) OECD. Test No. 201: Freshwater Alga and Cyanobacteria, Growth Inhibition Test; OECD Guidelines for the Testing of Chemicals, Section 2, OECD Publishing: Paris, France, 2011; http://dx.doi.org/10.1787/9789264069923-en.

(28) Mayer, P.; Cuhel, R.; Nyholm, N. A simple in vitro fluorescence method for biomass measurements in algal growth inhibition tests. Water Res. 1997, 31 (10), 2525-2531.

(29) Peijnenburg, W. J. G. M.; Baalousha, M.; Chen, J.; Chaudry, Q.; Von der kammer, F.; Kuhlbusch, T. A. J.; Lead, J.; Nickel, C.; Quik, J. T. K.; Renker, M.; Wang, Z.; Koelmans, A. A. A Review of the Properties and Processes Determining the Fate of Engineered Nanomaterials in the Aquatic Environment. Crit. Rev. Environ. Sci. Technol. 2015, 45 (19), 2084-2134.

(30) Quik, J. T. K.; van De Meent, D.; Koelmans, A. A. Simplifying modeling of nanoparticle aggregation-sedimentation behavior in environmental systems: A theoretical analysis. Water Res. 2014, 62, 193-201.

(31) Chen, W.; Duan, L.; Wang, L. L.; Zhu, D. Q. Adsorption of hydroxyl- and amino-substituted aromatics to carbon manotubes. Environ. Sci. Technol. 2008, 42 (18), 6862-6868.

(32) Jin, X.; Jiang, J.; Sheng, L.; Jin, M. Interspecies Competition between Microcystis aeruginosa and Scenedesmus obliquus under 
Phenanthrene Stress. Polish Journal of Environmental Studies 2014, 23

(5), 1609-1616.

(33) Zhu, X.; Kong, H.; Gao, Y.; Wu, M.; Kong, F. Low concentrations of polycyclic aromatic hydrocarbons promote the growth of Microcystis aeruginosa. J. Hazard. Mater. 2012, 237, 371375.

(34) Yang, K.; Xing, B. S. Desorption of polycyclic aromatic hydrocarbons from carbon nanomaterials in water. Environ. Pollut. 2007, 145 (2), 529-537.

(35) Smith, K. E. C.; Dom, N.; Blust, R.; Mayer, P. Controlling and maintaining exposure of hydrophobic organic compounds in aquatic toxicity tests by passive dosing. Aquat. Toxicol. 2010, 98 (1), 15-24. 